\title{
Revolving door respiratory patients: A rehabilitative perspective
}

\author{
Massimiliano Polastri', Lara Pisani2, Andrea Dell'Amore3, Stefano Nava² \\ 1 Medical Department of Continuity of Care and Disability, Physical Medicine and Rehabilitation, \\ University Hospital St. Orsola-Malpighi, Bologna \\ 2 Department of Clinical, Integrated and Experimental Medicine (DIMES), Respiratory and Critical Care Unit, \\ University Hospital St. Orsola-Malpighi, Bologna \\ 3 Department of Cardiac-Thoracic and Vascular Diseases, Unit of Thoracic Surgery, University Hospital \\ St. Orsola-Malpighi, Bologna, Italy
}

\begin{abstract}
Rehabilitation is an integral component of care for patients affected by either acute or chronic pulmonary diseases. The key elements of rehabilitation treatment for critical respiratory patients are as follows: weaning from mechanical ventilation, respiratory therapy, physical reconditioning, and occupational therapy. It should be noted that patients affected by pulmonary diseases are prone to hospital re-admission due to frequent exacerbations, especially in cases with more severe stages of chronic obstructive pulmonary disease. A periodical worsening of clinical conditions is common in asthma, acute respiratory distress syndrome survivors, obstructive sleep apnea syndrome, and pulmonary fibrosis, as well as in patients with severe neuromuscular diseases. These patients are often identified as "revolving door patients". Pulmonary patients are typically forced to maintain bed rest, or at least spend most of their waking hours dealing with mobility limitations, due to various pathological conditions including dyspnea, fatigue, and poor tolerance of movements. Alterations in mood are common in pulmonary patients who experience a decreased quality of
\end{abstract}

Corresponding author: Massimiliano Polastri, Medical Department of Continuity of Care and Disability, Physical Medicine and Rehabilitation, University Hospital St. Orsola-Malpighi, Via G. Massarenti 9, 40138 Bologna, Italy. Tel. +39.051.2144980 - Fax +39.051.0822185.

E-mail: gbptap1@gmail.com

Key words: Pulmonary rehabilitation; noninvasive ventilation; acute respiratory distress syndrome; chronic obstructive pulmonary disease; intensive care units.

Conflict of interest: The authors have no conflicts of interest to declare.

Received for publication: 23 May 2017

Accepted for publication: 2 August 2017

(C) Copyright M. Polastri et al., 2017

Tipografia PI-ME Editrice, Italy

Monaldi Archives for Chest Disease 2017; 87:857

doi: $10.4081 /$ monaldi.2017.857

This article is distributed under the terms of the Creative Commons Attribution Noncommercial License (by-nc 4.0) which permits any noncommercial use, distribution, and reproduction in any medium, provided the original author(s) and source are credited. life and limited social interactions. These negative emotional and cognitive aspects can be a major limitation to the provision of care, because to enhance and facilitate a degree of autonomy, the patient must be cooperative and pro-active.

Rehabilitation is an integral component of care for patients affected by either acute or chronic pulmonary diseases. The key elements of rehabilitation treatment for critical respiratory patients are as follows: weaning from mechanical ventilation, respiratory therapy, physical reconditioning, and occupational therapy. It should be noted that patients affected by pulmonary diseases are prone to hospital re-admission due to frequent exacerbations, especially in cases with more severe stages of chronic obstructive pulmonary disease (COPD). A periodical worsening of clinical conditions is common in asthma, acute respiratory distress syndrome (ARDS) survivors, obstructive sleep apnea syndrome (OSAS), and pulmonary fibrosis, as well as in patients with severe neuromuscular diseases (particularly the elderly). These patients are often identified as "revolving door patients".

At this time, rehabilitative requirements in pulmonary units are well-established, and there is a consensus among clinicians and healthcare professionals on the importance of early mobilization, particularly in respiratory intensive care units (RICUs) [1]. A patient that survives to the RICU will undergo demanding physiotherapy, and muscle wasting has been identified as one of the major clinical sequelae in intensive care unit (ICU) patients whose stay is longer than 7 days. In support of this, it has been found that rectus femoris crosssectional area decreased significantly from days 1 to 7 , and continued to decrease after day 10, in an ICU population; thus, leg protein breakdown is greater than protein synthesis [2]. Biomechanically, significant muscle wasting, particularly involving the lower body, may represent a negative predictor of recovery of ambulation and/or postural transfers (i.e., supine to sitting, sitting to standing). The quadriceps and gluteus function, as well as postural synergies, provide an upright position that is important for personal autonomy: muscle wasting can negatively affect muscular and proprioceptive abilities, resulting in lack of and/or delayed recovery of motor abilities. In other words, the recovery phase following an acute event or flare of a pulmonary condition is often characterized by the need for intensive mobility and respiratory programs to restore both physical function and respiratory efficiency, as well as to maximize the patient's autonomy.

Pulmonary patients are typically forced to maintain bed rest, or at least spend most of their waking hours dealing with mobility limitations, due to various pathological conditions including dyspnea, fatigue, and poor tolerance of movements. It should be noted that many 
pulmonary patients are subjected to overnight non-invasive ventilation treatments to prevent a worsening of gas exchange. However, this treatment can affect sleep quality. Pulmonary patients do not have the same requirements as patients that undergo thoracic surgery or lung transplantation: the latter often obtain improved mobility and autonomy postoperatively. Rehabilitation has been identified as a fundamental aspect of an enhanced recovery pathway for thoracic surgery patients [3]. In particular, a preoperative rehabilitation program is advisable for patients waiting for thoracic surgery who experience suboptimal or poor respiratory function. At the same time, postoperative rehabilitation is a crucial therapeutic intervention to achieve or accelerate the patient's return to normal daily activities. On the contrary, patients suffering from an acute onset of symptoms, or a flare of a pre-existing pulmonary disease, may require a more customized rehabilitative intervention, avoiding activities that worsen the dyspnea and fatigue. In this regard, it should be noted that ARDS (the effects of which persist for at least 12 months) survivors can experience cognitive impairment (with a prevalence of 70\%), depression, anxiety, and decreased quality of life (significantly lower than the normal population) [4].

These negative emotional and cognitive aspects can be a major limitation to the provision of care, because to enhance and facilitate a degree of autonomy, the patient must be cooperative and pro-active. Alterations in mood are common in pulmonary patients who experience a decreased quality of life and limited social interactions. Considering these factors, establishing a rehabilitative intervention can be challenging in pulmonary departments if appropriate physiotherapist staff are not available. This is particularly important for severely obese subjects (body mass index $>60$ ) affected by OSAS. Understaffing in hospitals can cause accidents and represents a source of inefficiency [5]. It has been found that understaffing is an important barrier to providing care in ICUs and RICUs [6,7]. On the other hand, economic constraints are used to justify inappropriate personnel allocation, or to mask low or insufficient investment in public health services. As previously highlighted, pulmonary patients are recurrent patients, as shown in Figure 1; thus, it is important to decrease re-admission rates in these populations. This requires high standards of care and an appropriate rehabilitation program, which should be used to educate and instruct patients on the management of home care. Indeed, patients with pulmonary diseases are subjected to rehabilitative therapeutic treatment once they are discharged from hospital facilities: this often represents a major barrier to the continuum of care. The high rate of clinical worsening among pulmonary patients must be considered as one of the most important factors when planning rehabilitation. Decreasing hospital re-admission rates, which are high in patients with respiratory impairment caused by pulmonary pathological conditions, could be challenging and expensive if the "recurrence factor" is neglected. There is not enough attention on the difficulties encountered by professionals when providing rehabilitation in pulmonary units. From a rehabilitative perspective, pulmonary patients are very likely to require a more demanding treatment regimen when compared with other patient populations (e.g., surgical patients).

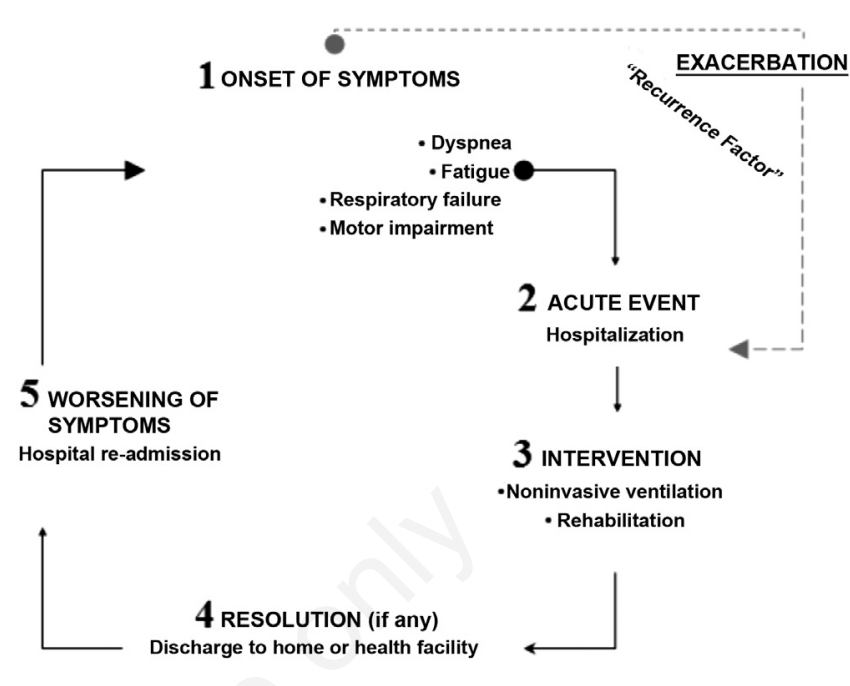

Figure 1. A typical flow diagram of pulmonary patients.

\section{References}

1. Clini E, Ambrosino N. Early physiotherapy in respiratory intensive care unit. Respir Med 2005;99:1096-104.

2. Puthucheary ZA, Rawal J, McPhail M, et al. Acute skeletal muscle wasting in critical illness. JAMA 2013;310:1591-600.

3. Scarci M, Solli P, Bedetti B. Enhanced recovery pathway for thoracic surgery in the UK. J Thorac Dis 2016;8:S78-S83.

4. Hopkins R0, Weaver LK, Collingridge D, et al. Two-year cognitive, emotional, and quality-of-life outcomes in acute respiratory distress syndrome. Am J Respir Crit Care Med 2005;171:340-7.

5. Polastri M. Are we sufficiently aware of safety in rehabilitation? Int J Ther Rehabil 2013;20:426-7.

6. Malone D, Ridgeway K, Nordon-Craft A, et al. Physical therapist practice in the intensive care unit: results of a national survey. Phys Ther 2015;95:1335-44.

7. Polverino E, Nava S, Ferrer M, et al. Patients' characterization, hospital course and clinical outcomes in five Italian respiratory intensive care units. Intensive Care Med 2010;36:137-42. 